\title{
El problema del consumo de cannabis: el papel del Sector Salud
}

\author{
Jesús del Bosque, ${ }^{1}$ Carmen Fernández, ${ }^{2}$ Ricardo Sánchez Huesca, ${ }^{2}$ David Bruno Díaz, ${ }^{2}$ \\ Alma Delia Gutiérrez López, ${ }^{2}$ Alba Fuentes Mairena, ${ }^{3}$ Mariana Espínola, ${ }^{4}$ Noé González García, ${ }^{5}$ \\ Arturo Loredo Abdalá, ${ }^{5}$ Ma. Elena Medina-Mora, ${ }^{6}$ Ricardo Nanni Alvarado, ${ }^{6}$ Guillermina Natera, ${ }^{6}$ \\ Tania Real, ${ }^{6}$ Raúl Sansores, ${ }^{7}$ Oscar Prospéro-García, ${ }^{7}$ Juan Zinser, ${ }^{8}$ Cosme Suárez, ${ }^{9}$ Angélica Beltrán 9
}

Concenso

\begin{abstract}
ANTECEDENTES
Un grupo de investigadores y clínicos de diversas especialidades, reunidos por la Coordinación de Institutos Nacionales de Salud y Hospitales de Alta Especialidad, ha analizado en forma conjunta los principales problemas de adicciones que aquejan al país con el fin de identificar campos para la colaboración interinstitucional y hacer propuestas de política pública. Este grupo previamente publicó un documento sobre el abuso de bebidas con alcohol y en esta ocasión abordará el tema del consumo de Cannabis. El grupo de trabajo identificó el consumo de esta sustancia como segunda prioridad en la problemática del consumo de drogas en nuestro país.
\end{abstract}

\section{INTRODUCCIÓN}

La Cannabis ha sido objeto de importante debate internacional. Algunas voces que proponen la legalización suponen que esta medida quitaría ganancias a los traficantes y existe consenso de que su consumo no debe de ser motivo de encarcelamiento. Algunos grupos promueven su uso medicinal, otros más reconocen algunos beneficios potenciales para la salud pero no documentan los riesgos de la Cannabis fumada, de ahí que piensen que la alternativa será usar medicamentos que contienen el principio activo (THC), cuyos beneficios para la salud estén por encima de los daños que ocasionen.

Estos argumentos que llegan a nuestros jóvenes no indican que la Cannabis sea una droga segura. Si bien cuando se le compara con otras drogas tiene menor nivel de toxicidad y se le asocia con menos consecuencias sociales éstas sí están presentes como cuando se usa al conducir automóviles. Existe evidencia de que a mayor nivel de uso mayor es la probabilidad de que se usen otras drogas. ${ }^{1}$ Durante la intoxicación se ve alterada la memoria de corto plazo, la atención, el juicio y otras funciones cognitivas; también afecta la coordinación y el balance y puede aumentar el ritmo cardiaco. A largo plazo, en usuarios crónicos, existe evidencia de la ocurrencia de déficits cognitivos más duraderos, reversibles después de la abstinencia mantenida; también se ha asociado con mayor riesgo de cáncer, depresión y psicosis. La exposición in utero deja secuelas a largo plazo. Por ejemplo, la evidencia de que durante la adolescencia hay un peor desempeño en tareas que requieren memoria visual, análisis e integración en quienes fueron expuestos durante la gestación. ${ }^{2}$

Es por tanto un problema que debemos de atender con información basada en evidencia y con medidas que nos permitan apoyar el desarrollo sano de nuestros niños y jóvenes.

\section{Mecanismos neurobiológicos de la cannabis}

La cannabis sativa es una planta que aloja al Delta-9-Tetrahidrocanabinol (THC)que es su componente activo. ${ }^{3}$ En la cannabis sativa está presente en un 5\% y en el hachís en mayor contración, de $10 \%$ a $20 \%$; y con las modificaciones en su cultivo se ha logrado hasta una concetración del 19\% al 30\%. El THC alcanza al cerebro en unos cuantos minutos. Los efectos subjetivos los experimenta el usuario normalmente a los $30 \mathrm{~min}$. de haberla fumado. Se administra también por via oral y tópica. El THC se elimina en un periodo

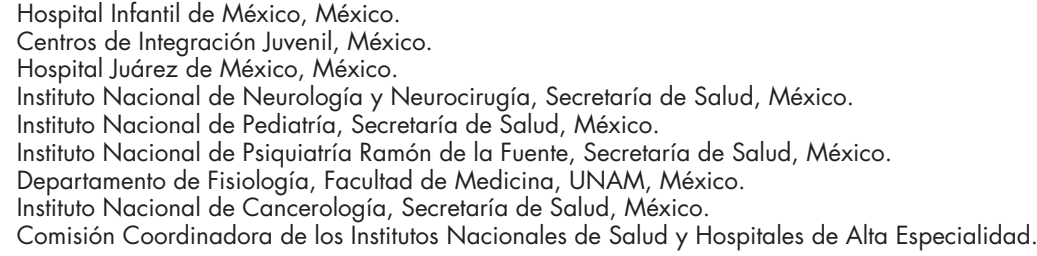


largo, de una a cuatro semanas. Los fumadores más experimentados son los que la eliminan más rápidamente. Los mecanismos de biodegradación son principalmente hepáticos, incluyendo el complejo de enzimas P-450. Finalmente, los metabolitos se eliminan por heces y orina.

La cannabis tiene efectos en cualquier sujeto que la consuma. Esto es debido a que el THC activa a un par de receptores que están en el Sistema Nervioso Central (SNC) y en diversas otras partes del organismo. Estos son los receptores canabinoides (CB) 1 y 2. El CB1 tiene una expresión privilegiada en el SNC; mientras que el CB2 la tiene en el sistema inmunológico.

Los endocanabinoides. En 1992 Mechoulam et al. describieron una molécula de naturaleza lipídica, aislada del cerebro del cerdo y que tiene propiedades semejantes a las de la cannabis. La llamaron anandamida. ${ }^{3}$ Dos años después se describió otro lípido al cual se nombró oleamida. ${ }^{4}$ Este también se une a los receptores de la cannabis (CB1 y CB2) y cuando se administra sistémicamente produce diversos efectos que son parecidos a los inducidos por el THC.

El receptor a canabinoides 1 (CB1). Este receptor está ampliamente distribuido en el cerebro, incluyendo el hipotálamo, la amígdala, el hipocampo, la corteza cerebral, el tallo cerebral y otras estructuras, y en éstas se localiza en las terminales de neuronas glutamatérgicas, colinérgicas, noradrenérgicas y GABAérgicas. La principal función del CB1 es reducir la probabilidad de liberación de estos neurotransmisores. ${ }^{5}$

Los endocanabinoides y el sistema de la motivación recompensa. Este sistema se encuentra en el cerebro y se compone primordialmente de dos estructuras: el núcleo accumbens (NAc) y el área tegmental ventral (ATV). ${ }^{3,6}$ El ATV activa al NAc al mismo tiempo que inactiva a la amígdala, núcleo involucrado en la generación de la sensación subjetiva de miedo y a la corteza prefrontal, área cortical ampliamente estudiada y reconocida como un componente crucial para la toma de decisiones. Así que los mecanismos de generación de la sensación subjetiva de placer prescinden activamente del miedo y del razonamiento. El mecanismo por medio del cual el ATV activa e inactiva las mencionadas estructuras es la liberación de dopamina. Los receptores D1 a la dopamina son excitadores, mientras que los D2 son inhibidores. Así, las neuronas del NAc expresan mayoritariamente D1 y las de la amígdala y corteza prefrontal, D2. Las terminales axónicas que llegan al ATV y al NAc expresan CB1, por lo que su aumento facilita la mayor activación de la neuronas de ambas estructuras y esto lleva al sujeto a experimentar la sensación subjetiva de placer. Es importante señalar que puede haber una predisposición genética para desarrollar dependencia a la cannabis. ${ }^{7}$ Su manifestación depende de la confluencia de factores de desarrollo con el contexto, tema que se aborda en el siguiente inciso.

Una observación adicional ilustra una de las razones por las que los adolescentes son más vulnerables a los efectos no sólo de la cannabis sino de cualquier sustancia de abuso. La corteza prefrontal, que como se indica arriba participa en la toma de decisiones, está inmadura en ellos. El proceso de maduración concluye hasta alrededor de los 30 años de edad. ${ }^{8}$ Así que los adolescentes toman decisiones considerando muy precariamente las consecuencias de su consumo.

Factores de riesgo psicosocial. La evidencia científica muestra que no todos los individuos están en el mismo riesgo de iniciar y desarrollar dependencia. Ésta se da por una combinación de factores que incrementan la vulnerabilidad y una ausencia de factores que protegen al individuo (por ejemplo supervisión de padres, alta autoestima, etc.). Los factores que aumentan la vulnerabilidad pueden ser biológicos y se relacionan con la sensibilidad a los efectos de la droga, los factores genéticos que marcan una predisposición de las personas a desarrollar dependencia a drogas si se encuentran con ellas, y factores ambientales que pueden incluir la exposición a drogas y la experiencia de vicisitudes en la infancia, especialmente cuando se ha sido víctima de violencia. ${ }^{9}$

La vulnerabilidad se define como la probabilidad que tiene una persona para desarrollar una condición, ésta, a su vez, está determinada por la magnitud de los factores de riesgo intra e interpersonales, emocionales, cognitivos y sociales. Estos se definen como aquellas condiciones que anteceden al problema y que aumentan la probabilidad de que éste ocurra. Así, por ejemplo, el padecer un trastorno emocional en la infancia temprana y llegar a la adolescencia sin haber recibido tratamiento constituye un factor de riesgo para el abuso de sustancias al aumentar la probabilidad de que éste ocurra como un intento del individuo para enfrentar su problema emocional.

Existen factores de riesgo comunes a todas las drogas y específicos para la cannabis. Ésta es una de las drogas ilícitas de mayor consumo en el mundo y puede actuar como "puerta de entrada" al uso de otras sustancias. Un factor de muy alto riesgo es el tener relación con pares usuarios de ellas. ${ }^{10,11}$

Otros factores reconocidos son: aislamiento social, baja competencia social, baja asertividad, deficiente manejo de conflictos interpersonales, ausencia de actitudes prosociales y baja capacidad para afrontar situaciones adversas.

En el contexto familiar existe una correlación significativa entre el uso de drogas en los jóvenes y el consumo de sustancias y las conductas antisociales en miembros de la familia, además de una actitud familiar tolerante ante el consumo y/o un clima familiar de conflicto o de violencia y maltrato, incluyendo el abuso sexual. En el ámbito escolar se describen factores como: bajo rendimiento académico, ausentismo, la expulsión o el abandono de la escuela, escaso interés por el estudio y por las actividades escolares.

Otros factores que se han identificado como de riesgo son la baja percepción de riesgo al consumo y alta accesibilidad de la sustancia, así como actitudes y expectativas positivas respecto al consumo y bajo control conductual percibido 
frente a situaciones que favorecen el uso de sustancias, baja autoestima, desesperanza y otros síntomas depresivos más o menos severos son factores comúnmente asociados. ${ }^{12-17}$

La edad de mayor riesgo para el inicio del consumo de cannabis, así como de mayor riesgo para sus efectos, es la adolescencia y disminuye o cesa con cambios de vida como el inicio de actividades laborales en empleos de tiempo completo o el matrimonio. ${ }^{18-22}$

El Trastorno por Déficit de Atención coexiste con el uso de sustancias entre el 34\% y el 63\% de los pacientes. Algunos estudios parentales han sugerido que cuando hay adecuada intimidad, autonomía y comportamientos de cuidado y protección existe un menor uso de sustancias; de igual manera se encuentra menor consumo con estilos autoritarios y de mayor supervisión parental. ${ }^{23}$ Los medios de comunicación y las normas sociales favorables al uso de sustancias también juegan un papel determinante.

Estudios realizados en Centros de Integración Juvenil de nuestro país encontraron como factores predictores del abuso de drogas ilícitas: exposición a estresores escolares, trastornos depresivos, vinculación con pares consumidores, pobre control de impulsos y baja percepción de riesgo. Como factores predictores de dependencia se agregan la accesibilidad y la exposición a estresores familiares. ${ }^{24-26}$

Antecedentes epidemiológicos. La Encuesta Nacional de Adicciones reporta que la cannabis es una de las sustancias preferidas por la población. De hecho ha ocupado los primeros lugares de preferencia entre la población desde una medición hecha en 1988. Para la medición de 2008, ${ }^{27}$ su consumo aumentó de 3.5 a 4.2\%. Su incidencia acumulada alcanza $4.2 \%$. Los datos de la encuesta indican que los adolescentes entre 12 y 17 años tienen más probabilidad de usar drogas cuando están expuestos a la oportunidad de hacerlo que quienes ya han alcanzado la mayoría de edad. Además, presentan 69 veces más probabilidad de usar cannabis cuando se las ofrecen regalada que sus compañeros que no han estado expuestos.

El consumo de cannabis se inicia con más frecuencia en la adolescencia, la edad de inicio para el consumo de drogas es típicamente temprana, los datos indican que la mitad de los usuarios (55.7\%) se inicia antes de la mayoría de edad. Esto indica que enfrentan también importantes riesgos que obligan a pensar en mecanismos para protegerlos de los daños potenciales a la salud durante este periodo de sus vidas.

Por su parte, la más reciente encuesta con estudiantes de educación media del Distrito Federal ${ }^{28}$ señala que el uso de cannabis "alguna vez en la vida" registra una prevalencia de $11.4 \%$, un aumento de 2.6 puntos porcentuales en relación con lo registrado en 2006. El aumento es ligeramente más acusado en hombres, en quienes el consumo pasó de $11.2 \%$ en 2006 a $14.0 \%$ en 2009 , mientras que en las mujeres fue de $6.4 \%$ a $8.8 \%$.

Características de los usuarios de cannabis en centros de tratamiento especializados. En el caso de los usuarios de drogas solicitantes de tratamiento en Centros de Integración Juvenil, en el primer semestre de 2010 el uso de cannabis "al- guna vez en la vida" fue reportado por 8509 casos $(72.4 \%$ del total). Quienes refirieron haberla utilizado en los 30 días previos a su ingreso a tratamiento (4416 casos) presentan algunas características distintivas, comparados con quienes no la consumieron en este lapso.

Entre las diferencias significativas entre los usuarios activos de cannabis y el resto de la población atendida destaca una menor edad al ingresar a tratamiento (21.3 contra 24.6 años), una proporción más alta de hombres (7.9 hombres por cada mujer contra 3.4 hombres por cada mujer) y una menor edad de inicio del consumo de tabaco (14 vs. 14.8 años), alcohol (14.3 vs. 14.9 años) y drogas ilícitas (15.2 vs. 17.2 años). De igual modo, los usuarios de cannabis en el último mes refieren haber utilizado un mayor número de drogas ilícitas alguna vez en la vida (2.9 contra 2.1$)$, con un consumo más alto de depresores con utilidad médica (principalmente benzodiacepinas), alucinógenos, estimulantes y crack.

Los usuarios de cannabis en el último mes reportan también una alta proporción de uso combinado de sustancias (dos o más sustancias en un mismo día, incluye el uso de alcohol), con una diferencia de más de 17 puntos respecto al resto de la población comparada (65.2\% contra $48.0 \%$ ), así como mayor frecuencia de abuso en el consumo de drogas ilícitas (57.7\% de los usuarios actuales de cannabis utilizó drogas diariamente o cada tercer día, contra $37.1 \%$ con esta frecuencia de uso en el resto de la población). ${ }^{29}$

Consecuencias psicosociales del uso de cannabis. Las repercusiones que la cannabis tiene en la salud del individuo y la sociedad conducen a la necesidad de establecer políticas efectivas que reduzcan sus consecuencias, tales como la violencia, las enfermedades o los sufrimientos a los consumidores y sus familiares, pues desconocen los efectos que esta sustancia tiene en la vida de sus hijos, lo que ocasiona estrés y sentimientos negativos que pueden afectar su salud física y mental.

Dado que los efectos del consumo en los jóvenes, además de estar en riesgo de adquirir una adicción, incluyen consecuencias en la distorsión de la percepción, afectación de la memoria, del juicio y habilidades para aprender y dificultades para pensar y solucionar problemas, los efectos en la sociedad se derivan de estas consecuencias.

La investigación en otros países demuestra claramente que la cannabis tiene el potencial para causar problemas en la vida cotidiana o empeorar los problemas existentes de una persona, además de afectar su vida social y su estatus. Varios estudios asocian a trabajadores que fuman cannabis con mayores ausencias, tardanza, accidentes, reclamaciones de compensación y el volumen de trabajo. Los estudiantes que la fuman generalmente obtienen calificaciones más bajas y tienen menos probabilidad de graduarse de la escuela secundaria que sus compañeros que no la consumen.

Daño al producto in utero y al lactante por el consumo materno de cannabis. Cuando la mujer consume cannabis durante el embarazo o en el período de lactancia existe la posibilidad de dañar el cerebro del producto. 
En este contexto, la experiencia en animales de laboratorio es mayor que en los seres humanos.

La exposición del Sistema Nervioso Central (SNC) a la cannabis originó el concepto de "daño teratógeno del cerebro y del desarrollo neurológico". Ello ocurre porque el THC atraviesa la barrera hematoencefálica cuando ya existe el sistema endocanabinoide lo que vulnera el desarrollo del SNC. Esta agresión ocasiona trastornos en la neurogénesis, la diferenciación entre neuronas, la sinaptogénesis, la mielinización y la migración de la glía, entre otros. Aunque este daño no origina malformación del SNC sí genera anormalidades funcionales que habitualmente no se detectan en el recién nacido. Además, la canabis se elimina por la leche materna.

Los efectos de la droga no provocan una alteración fenotípica del funcionamiento del SNC como crisis convulsivas o franco retardo en el desarrollo psicomotor, pero el daño se expresa como hiperactividad, impulsividad, problemas en la atención, en la memoria, ansiedad, depresión y se asocia con delincuencia en la niñez o en la adolescencia, pero no en la vida adulta.

Otros problemas asociados son: déficit en el peso y en la talla así como alteración en los patrones del sueño del menor. Cuando el consumo supera a los 12 meses antes del embarazo aumenta de dos a cinco veces el riesgo para desarrollar rabdomiosarcoma, neuroblastoma, malformación urogenital y microcefalia.

Estos efectos pueden ser modulados por ciertos factores como el tiempo de exposición de la madre a la droga, la dosis y la vía de contacto así como su asociación con otras sustancias "lícitas" e "ilícitas". 30-33

Comorbilidad psiquiátrica. Algunos estudios correlacionan el consumo de cannabis y su potencial asociación con psicopatología en algunos pacientes, lo cual se puede explicar desde dos perspectivas: 1. la hipótesis de la automedicación (en la que el sujeto trata de mejorar sus síntomas psiquiátricos con la cannabis) y 2. la teoría que postula que la sustancia es la causa o factor predisponente de la psicosis. En este último caso pueden presentarse tres situaciones: a) pacientes con carga genética predisponente que en presencia de la sustancia manifestarían fenotípicamente una psicosis; b) pacientes sin carga genética predisponente, en los que las sustancias per se podrían inducir psicosis y c) personas vulnerables que sometidas repetidamente al uso de la sustancia pueden desarrollar una psicosis por un fenómeno de sensibilización progresiva. En cualquiera de las posibilidades se asocian factores como: adultos jóvenes, sexo masculino, desempleo, bajo nivel educativo, historia de personalidad esquizoide, paranoide o esquizotípica, trastornos de conducta y dinámica familiar conflictiva.

Por otro lado, en relación a la automedicación se estima que en los pacientes con esquizofrenia el riesgo de presentar un trastorno por uso de sustancias a lo largo de la vida es 4.6 veces el de la población general, con una probabilidad de cinco veces para la cannabis.
Cuadro 1. Consumo de cannabis y salud mental

\begin{tabular}{ll}
\hline Autor & \multicolumn{1}{c}{ Asociación } \\
\hline $\begin{array}{l}\text { McKay y Tennant (2000) } \\
\text { Hall y Degenhardt (2000) }\end{array}$ & $\begin{array}{l}\text { Precipita el inicio o recaída de la es- } \\
\text { quizofrenia en sujetos predispuestos y } \\
\text { exacerba los síntomas. } \\
\text { Incrementa } 6 \text { veces el riesgo de esqui- } \\
\text { zofrenia en grandes consumidores ado- } \\
\text { Zammit (2002) }\end{array}$ \\
lescentes. \\
Lovasso (2001) \\
Les síntomas depresivos son 4 veces más \\
frecuentes en consumidores de cannabis.
\end{tabular}

Se ha reportado además la relación entre el consumo de cannabis, la psicosis y la depresión, pudiendo su consumo precipitar cuadros psicóticos agudos e incrementar el riesgo de esquizofrenia crónica en personas vulnerables, exacerbando por lo general los síntomas. Si bien algunos estudios muestran que el uso de cannabis durante la adolescencia incrementa el riesgo de padecer esquizofrenia, es difícil establecer una causalidad directa con la esquizofrenia (cuadro 1).

Lo que sí tiene sustento suficiente es la presencia del Síndrome Amotivacional (caracterizado por apatía, adinamia, anergia, desgano, postergación de toma de decisiones y de realización de actividades, es decir, la procrastinación; así como falta de conciencia de enfermedad y por ello no se acude a solicitar atención médica) que padecen los sujetos, secundario a su consumo crónico y en población susceptible, lo cual impacta significativamente en las diversas áreas de la vida del individuo y de sus familias. ${ }^{33-41}$

\section{Las indicaciones médicas de la cannabis}

El análisis y evaluación de los potenciales usos médicos de la cannabis tienen fundamentos científicos ya que en el organismo existen receptores cannabinoides $\left(\mathrm{CB}_{1}\right.$ y $\left.\mathrm{CB}_{2}\right)$ sobre los que actúan los diferentes componentes de la sustancia, como ya se mencionó. En diversas enfermedades se ha identificado la participación y disregulación de dichos receptores, por lo que su modulación tiene potenciales beneficios terapéuticos.

La cannabis contiene más de 60 fitocannabinoides de los cuales el THC es el más abundante. Del resto de los cannabinoides los más estudiados son el cannabinol (CBN) que es producto de la oxidación del THC y contiene el 10\% de su efecto, el cannabidiol (CBD) que no tiene efecto psicoactivo 
El problema del consumo de cannabis: el papel del Sector Salud

Cuadro 2. Estado del uso médico de cannabinoides en presentación farmacéutica

\begin{tabular}{|c|c|c|}
\hline Indicación & Efecto & Status de aprobación \\
\hline $\begin{array}{l}\text { Náusea y vómito secundario a } \\
\text { quimioterapia }\end{array}$ & $\begin{array}{l}\text { No agrega beneficio a lo conven- } \\
\text { cional }\end{array}$ & $\begin{array}{l}\text { Sí, en algunos países como } \\
\text { cuarta línea de tratamiento }\end{array}$ \\
\hline Anorexia secundaria a SIDA & & Sí \\
\hline Anorexia secundaria a cáncer & No es superior al acetato de megestrol & No \\
\hline Anorexia nervosa & No parece tener actividad & No \\
\hline Esclerosis múltiple & $\begin{array}{l}\text { Mejora espasticidad y dolor neuro- } \\
\text { pático }\end{array}$ & Sí \\
\hline Esclerosis lateral amiotrófica & $\begin{array}{l}\text { Mejora apetito y sueño pero no ca- } \\
\text { lambres y fasciculaciones }\end{array}$ & No \\
\hline Lesión espinal & Mejoría en dolor y espasticidad & No \\
\hline Epilepsia & $\begin{array}{l}\text { Puede disparar o suprimir convulsio- } \\
\text { nes. En la mayoría sin efecto }\end{array}$ & No \\
\hline Dolor secundario a cáncer & $\begin{array}{l}\text { Equivalente a codeína con más efec- } \\
\text { tos secundarios }\end{array}$ & $\begin{array}{l}\text { Sí, junto con analgésicos y al- } \\
\text { gunas restricciones }\end{array}$ \\
\hline Dolor neuropático & & $\begin{array}{l}\text { Sí, con analgésicos en algunos } \\
\text { países en cuarta línea }\end{array}$ \\
\hline Hiperactividad vesical & Mejora la urgencia e incontinencia & Sí \\
\hline Glaucoma & $\begin{array}{l}\text { No es superior a tratamientos con- } \\
\text { vencionales }\end{array}$ & No \\
\hline $\begin{array}{l}\text { Artritis reumatoide, enferme- } \\
\text { dad de Parkinson, enfermedad } \\
\text { de Crohn, psoriasis y urticaria }\end{array}$ & Posible beneficio & Requiere más investigación \\
\hline
\end{tabular}

pero si antiinflamatorio, analgésico, antipsicótico, antiisquémico, ansiolítico y antiepiléptico, y el cannabigerol (CBG) con efectos terapéuticos potenciales en las psicosis, la epilepsia, la ansiedad, las alteraciones del sueño, los procesos neurodegenerativos, la isquemia, la emesis, la ingesta de alimentos, la diabetes tipo I, la osteogénesis y el cáncer. A pesar de la larga lista de beneficios potenciales, éstos han sido difíciles de evaluar ya que muchos de ellos son bifásicos, con mayor actividad de manera aguda y a dosis bajas y disminución de la misma con incremento en las dosis y el uso crónico.

Estudiar el efecto terapéutico de la cannabis fumada o ingerida no es posible ni correcto por tres razones: 1 . dependiendo de la planta la concentración de cannabinoides es distinta con un rango de THC del 1 al 30\%, aunque en promedio es de aproximadamente el 10\%; 2. la forma de fumar es muy variable, tanto en la profundidad de la inhalación como en la frecuencia de la misma y 3. el humo de la cannabis contiene muchos de los carcinógenos del tabaco y por la forma de fumarla se retiene en los pulmones tres veces más alquitrán y cinco veces más monóxido de carbono. Por lo tanto, para evaluar médicamente la actividad de los cannabinoides se requiere de presentaciones elaboradas por la industria farmacéutica con dosis fijas, habitualmente bajas y que eviten los efectos tóxicos. Los siguientes medicamentos se han aprobado en algunos países, en ciertos casos con restricciones: Marinol (dronabinol tabletas de $2.5 \mathrm{mg}$ ), Sativex (spray oral THC 2.7mg + CBD 2.5mg por disparo) y Cesamet (nabilona tabletas de $1 \mathrm{mg}$ ).
En el cuadro 2 se presenta el estado del uso médico de las presentaciones farmacéuticas de cannabinoides en otros países.

Como puede observarse, las indicaciones médicas de los cannabinoides son mínimas y ninguna es insustituible por otros medicamentos. Aunque no se presentaron las diversas dosis que se han utilizado, en general son bajas (una tableta de marinol de $2.5 \mathrm{mg}$ equivale a una inhalación de cannabis fumada). Por ejemplo, la dosis en la anorexia secundaria al SIDA es de dos tabletas al día.

El uso de estos medicamentos debe vigilarse cuidadosamente y no administrarse a menores de 18 años, a personas con antecedentes de hipersensibilidad o problemas psiquiátricos, en particular esquizofrenia, en enfermedad hepática, renal y cardiopulmonar, en el embarazo, la lactancia ni en hombres con planes de fecundación. Debe vigilarse también la asociación con otros medicamentos ya que su efecto puede modificarse: asociados con el alcohol pueden aumentar el efecto sedante de este último. Hay que recordar que una inhalación de cannabis fumada puede producir niveles en sangre de THC cuyo efecto en la coordinación para conducir automóviles puede ser semejante al de $0.05 \%$ de alcohol (2-3 copas).

En resumen, los efectos fisiológicos y médicos de los cannabinoides pueden ser múltiples ya que existen receptores distribuidos en todo el cuerpo. Desde hace muchos años esto ha despertado el interés de la comunidad científica por su estudio e investigación. Aunque limitadas, se han establecido algunas indicaciones médicas para el uso de cannabinoides utilizando las presentaciones farmacéuticas 
Cuadro 3. Daños pulmonares y extrapulmonares asociados al consumo de cannabis

\begin{tabular}{ll}
\hline Pulmonares & \multicolumn{1}{c}{ Extrapulmonares } \\
\hline Síntomas respiratorios & Alteraciones orales \\
Bronquitis crónica & Cáncer \\
$\begin{array}{l}\text { Función pulmonar y limitación } \\
\text { del flujo aéreo }\end{array}$ & Accidentes automovilísticos \\
Cáncer de pulmón & Enfermedades cardiovasculares \\
Hiper-reactividad bronquial & Disfunción eréctil \\
Enfisema buloso & \\
Pneumotórax & \\
\hline
\end{tabular}

existentes. Existen guías de prescripción que permiten utilizar estos medicamentos con razonable seguridad, situación que no es extrapolable al uso médico de la cannabis fumada ya que con facilidad se puede exceder la dosis aprobada y el humo contiene, además, muchas sustancias tóxicas. ${ }^{42}$

Daños a la salud asociados al consumo de cannabis. El uso prolongado puede llevar a la adicción. Cuando las personas llegan a este punto la droga interfiere en muchos aspectos de su vida pero no la pueden dejar aunque quieran hacerlo. El riesgo es mayor para quienes inician el uso en la adolescencia, cuando el cerebro aún no ha madurado. Quienes desarrollan dependencia a esta sustancia pueden presentar un síndrome de abstinencia similar al que se experimenta cuando se deja de fumar. También pueden experimentar irritabilidad, dificultades de sueño, un deseo que no pueden controlar de consumir y ansiedad, y pueden volverse más agresivos durante este período.

Los daños a la salud asociados al consumo de cannabis no se han asumido de manera precisa porque se piensa que el consumo es menor que el de nicotina y porque se cree que es menor el número de consumidores. También es probable que exista la creencia de que el daño a la salud es menor que el provocado por el consumo de los productos derivados del tabaco. Además, es posible que su uso tenga más riesgos asociados a los estilos de consumo. Por ejemplo, los fumadores de cannabis, como ya se hizo mención, hacen aspiraciones más profundas y detienen más tiempo el humo en sus pulmones. La forma como los consumidores la empacan en el papel que utilizarán para fumar, hace que sea más la cantidad de hierba que la contenida en un cigarrillo de tabaco. Nunca usan filtros, lo que implica que la inhalación del contenido sea mayor que con el tabaco. Estos factores propician que la concentración de sustancias potencialmente tóxicas sea mayor que la que teóricamente ocurre con el cigarrillo.

Los daños a la salud asociados al consumo de cannabis se pueden dividir en pulmonares y extrapulmonares (cuadro 3).

\section{Daños al sistema respiratorio}

Síntomas respiratorios. Los fumadores de cannabis tienen más síntomas respiratorios que los no fumadores. En un estudio,
Cuadro 4. Síntomas respiratorios

\begin{tabular}{lcccc}
\hline & $\begin{array}{c}\text { Sólo } \\
\text { cannabis }\end{array}$ & $\begin{array}{c}\text { Cannabis } \\
\text { + tabaco }\end{array}$ & $\begin{array}{c}\text { Sólo } \\
\text { tabaco }\end{array}$ & $\begin{array}{c}\text { No } \\
\text { fumadores }\end{array}$ \\
\hline Silbidos & $26.7 \%$ & $34.1 \%$ & $30.4 \%$ & $11.1 \%$ \\
Tos & $29.3 \%$ & $28.6 \%$ & $40.2 \%$ & $4.9 \%$ \\
Opresión & $49.3 \%$ & $53.9 \%$ & $40.0 \%$ & $34.6 \%$ \\
en el pecho & & & & \\
Bronquitis crónica & $18.7 \%$ & $30.8 \%$ & $13.0 \%$ & $2.5 \%$ \\
\hline (Modificado de Aldington, 2008) & & &
\end{tabular}

Aldington ${ }^{43}$ comparó a individuos que solamente fumaban cannabis versus individuos que la combinaban con tabaco; individuos que sólo fumaban tabaco e individuos que no fumaban. Observó que los fumadores de cannabis presentaron más sibilancias, tos, opresión del pecho y síntomas de bronquitis crónica que los no-fumadores. La frecuencia de estos síntomas es comparable a la que se observa en los individuos que solamente fuman tabaco, pero cuando los sujetos fuman tanto cigarrillos de nicotina como de cannabis, entonces tienen más síntomas que cualquiera de los dos en forma independiente (cuadro 4).

Disminución de la función pulmonar. Existe una relación dosis respuesta y la disminución de la relación VEF1/CVF en los fumadores de cannabis. Asimismo, se observa aumento de la resistencia de la vía aérea y de la capacidad pulmonar total, cambios que en conjunto sugieren que fumarla puede constituir un riesgo para desarrollar EPOC (enfermedad pulmonar obstructiva crónica). En el mismo estudio ${ }^{43}$ los investigadores determinaron la presencia de enfisema en un pequeño grupo de fumadores de cannabis. Estos datos también han sido observados por otros investigadores. ${ }^{44}$

Cáncer de pulmón. El tabaquismo es, con mucho, la causa número uno de cáncer pulmonar. Debido a que es difícil encontrar individuos que solamente fumen cannabis y nunca hayan fumado tabaco, los estudios que señalan una asociación entre el consumo de cannabis y cáncer de pulmón son escasos. Hay, sin embargo, elementos en el contenido de la cannabis y la forma de su consumo que hacen posible la asociación. Por ejemplo, la cannabis contiene el doble de hidrocarburos poliaromáticos con poder cancerígeno que el tabaco, lo que en combinación con el mayor tiempo que el humo permanece en el pulmón favorece la actividad de los productos carcinógenos en el pulmón. Nueva Zelanda es un país en donde los consumidores no suelen combinar la cannabis con el tabaco. Ahí se realizó un estudio de casos y controles ${ }^{45}$ en los que se pudo diferenciar claramente a los usuarios que solamente consumían cannabis de aquellos que sólo consumían tabaco y, como grupo control, se incluyeron a individuos que nunca habían fumado ni una ni el otro y a individuos que mezclaban los dos. Se encontró que el riesgo relativo de desarrollar cáncer de pulmón era 5.7 veces mayor en aquellos que consumían solamente cannabis. 
Hiperreactividad bronquial e hipersensibilidad alérgica. Un grupo de investigadores de España ${ }^{46}$ demostró que un porcentaje importante de usuarios de cannabis tienen pruebas de IgE y de reto bronquial positivas a cannabis. Coincidentemente este grupo mostró más síntomas respiratorios.

Enfisema buloso, pneumotórax y fibrosis pulmonar. Se han descrito reportes de estas entidades asociadas al consumo de cannabis. Aunque los casos están documentados, se requiere de más evidencia para determinar en forma categórica esta relación.

Los consumidores de cannabis tienen más síntomas respiratorios que una persona que no consume ningún tipo de cigarrillo. Algunos síntomas como la opresión del pecho es mayor cuando se consume tabaco y cannabis. El cuadro 4 muestra los síntomas en estas cuatro poblaciones. ${ }^{45}$

\section{Complicaciones no pulmonares}

Complicaciones orales. Se han asociado a diversas otras complicaciones, desde estados mentales alterados cuando se combinan con algunos anestésicos locales, ${ }^{47}$ hasta xerostomía, leukoedema y mayor probabilidad de candidiasis oral. ${ }^{48}$

Cáncer de cabeza y cuello. En un estudio de casos y controles $^{49}$ de 173 pacientes diagnosticados con carcinoma escamoso de cabeza y cuello y 176 sujetos libres de este cáncer, se encontró que los fumadores de cannabis tienen un riesgo alto de desarrollar tal tipo de neoplasia. Vale la pena subrayar que estos hallazgos persistieron después de realizar ajustes por tabaquismo, sexo, edad, raza, educación y otros confusores. Estos investigadores encontraron un efecto positivo de relación causal y de dosis. Es decir, en aquellos que consumían más y por más tiempo, el riesgo era mayor.

\section{Accidentes automovilísticos}

En una revisión se encontró que las complicaciones no pulmonares más importantes son los accidentes automovilísticos relacionados con el consumo agudo de cannabis. Como ya se mencionó, ésta afecta la percepción del tiempo y la distancia. Dos fumadas de un cigarrillo de cannabis puede tener un efecto sobre el Sistema Nervioso semejante al producido por niveles séricos de alcohol no permitidos. ${ }^{50}$

\section{Enfermedades cardiovasculares}

También se han descrito casos de infarto cardiaco y crisis hipertensivas asociadas al consumo de cannabis. ${ }^{50}$ Sin embargo se requiere contar con más evidencias para poderlo asegurar.

\section{Disfunción eréctil}

Un estudio reciente ha dirigido su atención a los efectos potenciales de la cannabis sobre la salud sexual de los hombres. Sus datos sugieren que existe un efecto ${ }^{51}$ inhibitorio sobre la erección, pero se requiere de más investigación en este sentido para afirmarlo.

El consumo de cannabis va más allá del panorama social asociado al consumo de drogas ilícitas y de los efectos sobre la salud mental. De hecho, una serie de evidencias señalan que sus efectos, más allá de los descritos, pueden ser multisistémicos. Las razones por las que este daño no se ha hecho claro tienen que ver con el consumo oculto y la prohibición. El punto es más que importante porque en la medida que no se conozcan sus daños a la salud será más difícil hacer campañas de prevención utilizando argumentos de salud y no solamente los de la adicción que, per se, serían suficientes.

\section{Consideraciones respecto al tratamiento}

El verdadero reto dentro del manejo de las adicciones es el concerniente al mantenimiento del no consumo de forma constante y consistente a través del tiempo. Dentro de este apartado, la apetencia o craving por el consumo adquiere un valor primordial en el manejo psicofarmacológico, revistiendo relevancia el otorgar seguimiento continuo y permanente. Los objetivos de la fase de mantenimiento son: a) promover y mantener la sobriedad, b) en caso de no lograrlo, llevar a cabo la reducción del daño por medio de la ingesta moderada, c) auxiliar a generar alternativas para resolver situaciones críticas, d) corregir los factores precipitantes y asociados, e) tratar las complicaciones, f) aplicar estrategias preventivas de recaídas.

Como principios generales, el manejo de las adicciones debe considerar lo siguiente: ${ }^{52}$

1) No existe un único tratamiento adecuado para todas las personas,

2) Debe poder disponerse inmediatamente del tratamiento,

3) Un tratamiento efectivo contempla las múltiples necesidades del sujeto, no sólo su consumo de drogas,

4) El tratamiento individualizado y el plan de asistencia han de evaluarse continuamente y modificarse, si es necesario, para garantizar que el plan cubra las necesidades cambiantes de la persona,

5) Para la eficacia de un tratamiento es decisivo seguirlo durante un tiempo adecuado,

6) Los consejos (de forma individualizada o en grupo) y otras formas de terapia comportamental, son aspectos fundamentales para la eficacia de un tratamiento de adicción,

7) Para muchos pacientes los medicamentos constituyen un elemento importante del tratamiento, especialmente si se combinan con los consejos y otras terapias del comportamiento,

8) Aquellos sujetos adictos o con abuso de drogas que presentan simultáneamente una enfermedad mental, deben someterse a un tratamiento integral de ambos trastornos, 
9) La desintoxicación médica es sólo el primer paso del tratamiento de la adicción y, por sí sola, contribuye muy poco a modificar a largo plazo el hábito del consumo,

10) Un tratamiento no necesita ser voluntario para ser efectivo,

11) Hay que controlar continuamente la posibilidad de que exista consumo de drogas durante el tratamiento,

12) Los programas de tratamiento deben incluir la detección de enfermedades como el SIDA, las hepatitis B y $C$, la tuberculosis y otras enfermedades infecciosas e intentar ayudar a los pacientes para cambiar comportamientos con el fin de evitar que ellos mismos $u$ otras personas puedan infectarse,

13) La recuperación de la adicción a una droga puede ser un proceso muy largo y suele requerir múltiples periodos de tratamiento.

Otro punto fundamental es el abordaje psicoterapéutico en el cual es necesario aclarar y fundamentar el diagnóstico, fijar los objetivos a corto y a largo plazo, así como establecer los términos del contrato terapéutico. La participación de miembros de la familia del paciente es necesaria en el periodo inicial. En esta primera fase el objetivo es lograr el convencimiento del paciente de mantenerse en abstinencia.

Posteriormente se abordarán algunos de los factores que lo llevaron al consumo de las sustancias, ante esto el paciente debe aprender a manejar la ansiedad así como a integrar el abandono paulatino de los mecanismos psicológicos de defensa como la negación y la proyección, fortaleciendo asímismo la conciencia de su autocuidado. Es posible que se hagan evidentes problemas de tipo sexual, familiares $u$ otros, los cuales hay que abordar. Asímismo es menester auxiliarse de Grupos de Ayuda Mutua del tipo de Alcohólicos Anónimos y Narcóticos Anónimos para dar contención y reestructurar al paciente y reinsertarlo en los diversos ámbitos de su vida. Con la intervención anterior muchos pacientes terminan el tratamiento o se mantienen en abstinencia y continúan con el trabajo de identificación y resolución de los conflictos intrapsíquicos, al tiempo que realizan un inventario moral permanente de su actuar cotidiano que reparan diariamente, con lo cual ceden las áreas de conflicto y se reintegran a su sociedad.

Cabe señalar que siguiendo los lineamientos mencionados el pronóstico será favorable, sin embargo dentro de la evolución natural de la enfermedad está contemplada la reincidencia, siendo entonces la directriz terapéutica el espaciar dichos cuadros de reincidencia y que cada uno tenga menor duración hasta que remita totalmente.

Las intervenciones psicosociales deberán incluir los siguientes objetivos: a) establecimiento y mantenimiento de la alianza terapéutica, b) vigilancia del estado clínico del paciente, c) tratamiento de los estados de intoxicación y abstinencia, d) desarrollo y facilitación del cumplimiento de un programa terapéutico individualizado, e) prevención de las recaídas, f) educación sanitaria individual y familiar, g) reducción de la comorbilidad y de las secuelas del consumo y h) integración de las intervenciones realizadas con la coordinación de profesionales de otras disciplinas y de organizaciones no gubernamentales.

Consideraciones sobre el análisis de la legalización de la cannabis. El análisis para evaluar el proceso de legalización de las drogas es complejo ya que incluye aspectos médicos, económicos y múltiples situaciones sociales de carácter nacional e internacional. Sobre la legalización de la cannabis se han esgrimido argumentos de carácter médico y socioeconómicos, estos últimos en relación a la reducción del narcotráfico y las consecuencias derivadas del mismo. Los argumentos que defienden su legalización en base a sus propiedades medicinales tienen valor histórico, ya que en la actualidad vimos que todas las propiedades médicas que se le han conferido son cubiertas por medicamentos diversos y que no contienen cannabinoides. En los países que se tienen aprobadas algunas indicaciones médicas de los cannabinoides, éstas se basan en el uso de compuestos orales elaborados por la industria farmacéutica, como se ha señalado anteriormente, y no en la cannabis fumada, ya que el humo de la misma contiene muchos compuestos tóxicos $\mathrm{y}$, por otro lado, la concentración de THC que se requiere para obtener un efecto farmacológico es mínima y su concentración es muy variable de una planta a otra. El uso "recreativo" convencional que se le da a la cannabis por la gran mayoría de sus consumidores, rebasa en mucho los niveles sanguíneos compatibles con una indicación médica, alcanzando diferentes grados de toxicidad.

En cuanto al argumento de un posible beneficio en el control de su consumo, la evidencia internacional indica que esto no es esperable. En los EUA se ha estudiado, entre estudiantes de lo que equivale a tercero de preparatoria, cuál es el consumo de acuerdo a la percepción del riesgo. Entre 1975 y 2008 el consumo aumenta conforme disminuye la percepción del riesgo y viceversa. ${ }^{53}$ Esto permite suponer que, de legalizarse la cannabis en México, un fuerte mensaje que recibiría la juventud es que no es tan dañina.

El multimencionado argumento de la experiencia en otros países, principalmente Holanda, debe analizarse con cautela y de ninguna manera considerarse como un ejemplo a seguir. En Holanda se despenalizó en 1976 y su consumo, que ya había disminuido el año previo, se mantuvo estable durante siete años. Entre 1984 y 1996 el consumo se incrementó del 15 al 44\%, reduciéndose la cantidad permitida de 30 a 5 gramos. Inicialmente el uso se restringía a "coffee shops", en Amsterdam, que en 1980 eran un total de nueve. Estos aumentaron a 71 en 1985 y en 1997 había entre 1200 y 1500 en toda Holanda. ${ }^{54}$ Sobre el número de consumidores, en 2001 el 37\% de la población mayor de 12 años en Amsterdam la había probado y en poblaciones de menos de 500 domicilios, el 11\%. ${ }^{55}$ Con estos datos no se puede considerar que Holanda sea un buen ejemplo a seguir, particularmen- 
Cuadro 5. Referencias normativas en el campo de la cannabis

\begin{tabular}{|c|c|c|}
\hline Punitivo & $\begin{array}{c}\text { Derecho a la salud y obligaciones del } \\
\text { Estado Mexicano }\end{array}$ & Administrativa \\
\hline $\begin{array}{l}\text { En México no está penado el uso de dro- } \\
\text { gas (ciertas cantidades) sino el tráfico, } \\
\text { la producción (esto es, la manufactura, } \\
\text { fabricación, elaboración, preparación o } \\
\text { acondicionamiento de algún narcótico), } \\
\text { el transporte, el tráfico, el suministro gra- } \\
\text { tuito, la prescripción y el comercio (esto } \\
\text { es, vender, comprar, adquirir o enajenar } \\
\text { algún narcótico). También se imponen } \\
\text { penas a quienes aporten recursos o co- } \\
\text { laboren financieramente en los delitos } \\
\text { anteriores, a quienes siembren o permi- } \\
\text { tan que se siembre en terrenos de su po- } \\
\text { sesión alguna planta cuyo alcaloide esté } \\
\text { prohibido y realicen actos de publicidad } \\
\text { o propaganda para favorecer el consumo } \\
\text { de narcóticos. } \\
\text { Código Penal Federal Título VII capítulo } \\
\text { l: "Delitos contra la salud". Capítulo Vl: } \\
\text { substancias psicotrópicas. } \\
\text { Ley General de Salud, Capítulo 5: "Estu- } \\
\text { pefacientes". }\end{array}$ & $\begin{array}{l}\text { Constitución Mexicana, artículo } 4 . \\
\text { La Convención Única de } 1961 \text {, su Pro- } \\
\text { tocolo y el Convenio sobre Sustancias } \\
\text { Psicotrópicas. } \\
\text { La Convención de Viena de } 1988 . \\
\text { Ley General de Salud, regula: } \\
\text { 1. Promoción de la salud (artículo 73). } \\
\text { 2. La atención médica (en sus funciones } \\
\text { preventiva, curativa y rehabilitadora } \\
\text { a que se refiere el artículo 33). } \\
\text { 3. Las personas que usen habitualmente } \\
\text { estupefacientes o sustancias psicotró- } \\
\text { picas (artículo 74). } \\
\text { 4. Al programa contra la farmacode- } \\
\text { pendencia que evalúa el Consejo } \\
\text { Nacional contra las Adicciones. }\end{array}$ & $\begin{array}{l}\text { NORMA Oficial Mexicana NOM-028-SSA2-1999, } \\
\text { Para la prevención, tratamiento y control de las adic- } \\
\text { ciones. } \\
2.1 \text { NOM-001-SSA 1-1993, Que instituye el procedi- } \\
\text { miento por el cual se revisará, actualizará y editará la } \\
\text { Farmacopea de los Estados Unidos Mexicanos. } \\
2.2 \text { NOM-025-SSA2-1994, Para la prestación de servi- } \\
\text { cios de salud en unidades de atención integral hospita- } \\
\text { laria médico-psiquiátrica. } \\
2.3 \text { NOM-010-SSA2-1993, Para la prevención y con- } \\
\text { trol de la infección por virus de la inmunodeficiencia } \\
\text { humana. } \\
2.4 \text { NOM-009-SSA2-1993, Para el fomento de la salud } \\
\text { del escolar. 2.5 NOM-017-SSA2-1994, Para la vigi- } \\
\text { lancia epidemiológica. } 2.6 \text { NOM-168-SSA 1-1998, Del } \\
\text { expediente clínico. } \\
\text { Reglamento de Control Sanitario de Actividades, Es- } \\
\text { tablecimientos, Productos y Servicios destinado a los } \\
\text { actos de control sanitario de la fabricación de estupefa- } \\
\text { cientes y sustancias psicotrópicas, prescripción médica, } \\
\text { expendio, importación y exportación de unos y otras. }\end{array}$ \\
\hline
\end{tabular}

te si tomamos en cuenta que en México, en una edad semejante, el consumo es mucho menor. Y por otro lado, una gran diferencia entre Holanda y México es que nuestro país representa una vía de paso al país que consume la mayor cantidad de drogas en el mundo, por lo que un proceso de legalización en México no puede desarrollarse sin una estrecha vinculación con los Estados Unidos.

Síntesis del marco legal en materia de adicciones en México. El marco jurídico sobre las adicciones en nuestro país es amplio y responde a distintas dimensiones: a) punitivo, se refiere a las normas que regulan y castigan el tráfico, producción y distribución de diversas sustancias ilícitas, b) derecho a la salud y obligaciones del Estado Mexicano, se refiere a las obligación del Estado para garantizar el acceso a la salud de todo ciudadano que sufre alguna adicción y las obligaciones asumidas en el ámbito internacional, c) administrativa, todo tipo de reglamentación sobre el manejo de sustancias que pueden producir adicciones pero cuyo uso es lícito y sobre la regulación de los establecimientos que dan tratamiento. Así, el marco legal lo componen la Constitución Política de los Estados Unidos Mexicanos, los tratados internacionales ratificados por nuestro país, leyes federales, leyes estatales, reglamentos, decretos, acuerdos y normas oficiales mexicanas.

El cuadro 5 resume parte de la legislación.

La cannabis no es una droga inocua, tiene consecuencias negativas en la salud y no existen elementos que justifiquen su legalización con fines de uso médico. La medida podría tener más consecuencias negativas que positivas en cuanto a salud y calidad de vida de niños, adolescentes y jóvenes, por la etapa de crecimiento y la suceptibilidad neuronal que varía en los individuos. La legalización facilitaría el acceso a la droga, transmitiéndole a la población el mensaje de un menor riesgo; lo que a su vez se ha asociado a un mayor consumo.

\section{REFERENCIAS}

1. Babor T, Miller P, Edwards G. Vested interests, addiction research and public policy. Addiction 2010;105(1):4-5.

2. National Institute on Drug Abuse (NIDA). Abuso de la marihuana. Disponible en línea: http://www.nida.nih.gov/ResearchReports/Marihuana/default.html (recuperado en 2011).

3. Méndez M, Ruiz AE, Prieto B, Romano A et al. El cerebro y las drogas, sus mecanismos neurobiológicos. Salud Mental 2010;33:451-456.

4. Cravatt DF, Prospéro-García O, Siuzdak G, Gilula NB, et al. Chemical characterization of a family of brain lipids that induce sleep. Science 1995;268:1506-1509.

5. Di Marzo V, Bifulco M, De Petrocellis L. The endocannabinoid system and its therapeutic exploitation. Nature Rev. Drug Discovery 2004:3:771-784.

6. Koob GF, Volkow ND. Neurocircuitry of addiction. Neuropsychopharmacology Reviews 2010;35:217-238.

7. Ruiz AE, Méndez M, Prieto Gómez B, Romano A et al. El cerebro, las drogas y los genes. Salud Mental 2010;33:535-542.

8. Dumontheil I, Burgess PW, Blakemore S-J. Development of rostral prefrontal cortex and cognitive and behavioral disorders. Dev Med Child Neurol 2008;50:168-181.

9. Benjet C, Borges G, Méndez E, Fleiz C, Medina-Mora ME. The association of chronic adversity with psychiatric disorder and disorder severity in adolescents. Eur Child Adolesc Psychiatry 2011;20(9):459-468.

10. Fergusson D, Boden J, Horwood L. Cannabis use and other illicit drug use: testing the cannabis gateway hypothesis. Addiction 2006;101(4):556-569. 
11. Fergusson D , Boden J, Horwood L. The developmental antecedents of illicit drug use: Evidence from a 25-year longitudinal study. Drug Alcohol Depend 2008;96(1-2): 165-177.

12. Arellanez J, Diaz D Wagner F, Pérez V. Factores psicosociales asociados con el abuso y la dependencia de drogas entre adolescentes: Análisis bivariados de un estudio de casos y controles. Salud Mental 2004;27(3):54-64.

13. Rodríguez S, Diaz B, Gracia S, Guerrero A. Capacidad predictiva de la Teoría de la Conducta Planificada en la intención y uso de drogas ilícitas entre estudiantes mexicanos. Salud Mental 2007;30(1):68-81.

14. Newman K, Harrison L, Dashiff C, Davies S. Relationships between parenting styles and risk behaviors in adolescent health: an integrative literature review. Rev Lat Am Enfermagem 2008;16(1):142-150.

15. Lloyd DA, Turner RJ. Cumulative life-time adversities and alcohol dependence in adolescence and young adulthood. Drug Alcohol Depend 2008;93:217-226.

16. Sartor C, Lynskey M, Bucholz K, McCutcheon V et al. Childhood sexual abuse and the course of alcohol dependence development: Findings from a female twin sample. Heath Drug Alcohol Depend 2007;89(2-3):139-144.

17. Kwok-Kei Mak, Sai-Yin Ho, Neil Thomas G, Schooling CM et al. Family structure, parent-child conversation time and substance use among Chinese adolescents. BMC Public Health 2010;10:503.

18. Lynskey M, Vink J, Boomsma D. Early onset cannabis use and progression to other drug use in a sample of Dutch twins, Behavior Genetics 2006;36(2):195-200.

19. Russell K, Dryden DM, Yuanyuan Liang, Friesen C et al. Risk factors for methamphetamine use in youth: a systematic review. BMC Pediatrics 2008;8:48 doi:10.1186/1471-2431-8-48.

20. Bretteville-Jensen A, Melberg H, Jones A. Sequential patterns of drug use initiation, Can we relieve in the gateway theory? BE J Econom Analysis Policy 2008;8(2): 1846-1846.

21. Wu B, Mao ZF, Rockett IR, Yue Y. Socioeconomic status and alcohol use among urban and rural residents in China. Subst Use Misuse 2008;43(7):952-966.

22. Chuan-Yu Chen, Storr C, Anthony J. Early-onset drug use and risk for drug dependence problems. Addict Behav 2009;34(3):319-322.

23. McLaughlin K, Greif Green J, Gruber M, Sampson N et al. Childhood adversities and adult psychopathology in the National Comorbidity Survey Replication (NCS-R) II: Associations with persistence of DSM-IV disorders. Arch Gen Psychiatry 2010;67(2):124-132.

24. Diaz N, Arellanez J, Martínez J. Uso de drogas y factores psicosociales asociados entre estudiantes de educación media básica del estado de Nuevo León. En: Secretaría de Salud, CONADIC. Observatorio mexicano en tabaco, alcohol y otras drogas. México: SSA, CONADIC. 2002; pp. 133-136.

25. Diaz N, García R. Factores psicosociales de riesgo de consumo de drogas ilícitas en una muestra de estudiantes mexicanos de educación media. Rev Panam Salud Pública 2008;24(4):223-232.

26. Sánchez H, Guisa V, Ortiz R, de Leó G. Detección temprana de factores de riesgo para el consumo de sustancias ilícitas. Salud Mental 2002;25(3):1-11.

27. Secretaría de Salud. Encuesta Nacional de Adicciones 2008. México: Instituto Nacional de Salud Pública; 2008.

28. Villatoro J, Gaytán F, Moreno M, Gutiérrez ML et al. Consumo de alcohol, tabaco y otras drogas en la ciudad de México. Medición 2009. México: Instituto Nacional de Psiquiatría Ramón de la Fuente Muñiz; 2010.

29. Sistema de Información Epidemiológica del Consumo de Drogas. SIECD (2011). Consumo de drogas en pacientes de primer ingreso a tratamiento en Centros de Integración Juvenil, enero-junio de 2010. México: Centros de Integración Juvenil; 2011.
30. English DR, Hulse GK, Milne E, Holman CD. Maternal cannabis use and birth weight: A meta-analysis. Addiction 1997;92:1553-1560.

31. Rivkin MJ, Davis PE, Lemaster JI, Cabral HJ. Volumetric MRI study of brain in children with intrauterine exposure to cocaine, alcohol, tobacco and marijuana. Pediatrics 2008;121:741-750.

32. Campolongo P, Trezza V, Ratano P, Palmery M. Developmental consequences of perinatal cannabis exposure: behavioral, and neuroendocrine effects in adult roedents. Psychopharmacology 2011;214:5-15.

33. Day N, Leech S, Goldschmidt L. The effects of prenatal marijuana exposure on delinquent behaviors are mediated by measures of neurocognitive functioning. Neurotoxicology Teratology 2011;1:129-136.

34. McKay DR, Tennant CC. Is de grass greener? The link between cannabis and psychosis. Med J Aust 2000;172:284-286.

35. Hall W, Degenhardt L. Adverse health effects of non-medical cannabis use. Lancet 2009;17:(9698):1383-1391.

36. Andreasson S, Allebeck P, Engstrom A, Rydberg U. Cannabis and schizophrenia. A longitudinal study of Swedish conscripts. Lancet 1987;2:1483-1486.

37. Zammit S, Allebeck P, Andreasson S, Lundberg I et al. Self reported cannabis use as a risk factor for schizophrenia in Swedish conscripts of 1969: historical cohort study. Br Med J 2002;325:1199-1201.

38. Bovasso GB. Cannabis abuse as a risk factor for depressive symptoms. Am J Psychiatry 2001;158:2033-2037.

39. Rey J. Cannabis and mental health. Br Med J 2002;325: 1183-1184.

40. Van Os J, Bak M, Hanssen M, Biji RV et al. Cannabis use and psychosis: A longitudinal population-based study. Am J Epidemiol 2002;156:319-327.

41. Arsenault L, Cannon M, Poulton R, Murray R et al. Cannabis use in adolescent and risk for adult psychosis: longitudinal prospective study. Br MedJ 2002;325:1212-1213.

42. Marihuana. Canada: Information for health care professionales; 2010.

43. Aldington S, Williams M, Nowitz M, Weatherall M et al. Effects of cannabis on pulmonary structure, function and symptoms. Thorax 2007;62:1058-1063.

44. Hancox RJ, Poulton R, Ely M, Welch D et al. Effects of cannabis on lung function: a population-based cohort study. Eur Respir J 2010;35:42-47.

45. Aldington S, Harwood M, Cox B, Weatherall M et al. Cannabis use and risk of lung cancer: a case-control study. Eur Respir J 2008;31:280-286.

46. Armentia A, Castrodeza J, Ruiz-Muñoz P, Martínez-Quesada J et al. Allergic hypersensitivity to cannabis in patients with allergy and illicit drug users. Allergol Immunopathol (Madr) 2011;39(5):271-279.

47. Cho CM, Hirsch R, Johnstone S. General and oral health implications of cannabis use. Aust Dent J 2005;50:70-74.

48. Versteeg PA, Slot DE, van der Velden U, van der Weijden GA. Effect of cannabis usage on the oral environment: a review. Int J Dent Hyg 2008;6:315-320.

49. Zhang ZF, Morgenstern H, Spitz MR, Tashkin DP et al. Marijuana use and increased risk of squamous cell carcinoma of the head and neck. Cancer Epidemiol Biomarkers Prev 1999;8:1071-1078.

50. Hall W, Degenhardt L. Adverse health effects of non-medical cannabis use. Lancet 2009;374(9698):1383-1391.

51. Shamloul R, Bella AJ. Impact of cannabis use on male sexual health. J Sex Med 2011;8:971-975.

52. National Institute on Drug Abuse (NIDA). Abuso de la marihuana. Disponible en línea: http://www.nida.nih.gov/ResearchReports/Marihuana/default.html

53. The Monitoring the Future study. Michigan: The University of Michigan; 2008.

54. MacCoun R, Reuter P. Interpreting dutch cannabis policy: Reasoning by analogy in the legalization debate. Science 1997;278:47-52.

55. Dolin B. National drug policy: The Netherlands. prepared for the senate special committee on illegal drugs. Law and Government Division; 2001.

Artículo sin conflicto de intereses 